Check for updates

Cite this: RSC Adv., 2021, 11, 3143

\title{
Synthesis of new pyridines with sulfonamide moiety via a cooperative vinylogous anomeric-based oxidation mechanism in the presence of a novel quinoline-based dendrimer-like ionic liquid $\dagger$
}

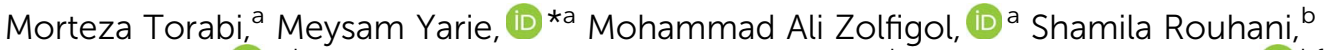 \\ Shohreh Azizi, (D) ${ }^{\mathrm{cd}}$ Temitope O. Olomola, ${ }^{e}$ Malik Maaza ${ }^{\mathrm{cd}}$ and Titus A. M. Msagati (D) bf \\ In the present study, we reported the synthesis of a novel quinoline-based dendrimer-like ionic liquid. After \\ characterization of the mentioned ionic liquid with suitable techniques such as Fourier transform infrared \\ spectroscopy (FT-IR), energy dispersive X-ray spectroscopy (EDX), elemental mapping, \\ thermogravimetric analysis (TGA) and derivative thermogravimetry (DTG), its catalytic performance was \\ investigated in the synthesis of new pyridines with sulfonamide moiety via a cooperative vinylogous \\ anomeric-based oxidation mechanism under mild reaction conditions. All target molecules were \\ achieved in short reaction times and high yields.
}

Received 4th November 2020

Accepted 30th November 2020

DOI: $10.1039 / \mathrm{d} 0 \mathrm{ra0} 0400 \mathrm{e}$

rsc.li/rsc-advances

characteristics have led to ILs becoming a hot research field for multidisciplinary scientists. ${ }^{12-15}$

The sulfonamide structural kernel (also known as sulfa drugs), is a prominent core in medicinal chemistry as compounds bearing this functional group exhibit varied pharmaceutical effects. For example, several bioactive molecules containing sulfonamide group were used as inhibitors of HIV protease, antibacterials, antibiotic, antitumor, antihypertensive, anticonvulsants and antifungal agents. The biological importance of sulfonamide containing drugs are well demonstrated in the literatures. ${ }^{16-25}$ Some sulfonamide drugs are portrayed in Scheme 1.

Another prominent structure core, which ubiquitous in biologically active compounds, is pyridine motifs. Pyridines have a key role in the biological behavior of several natural molecules and also, they are responsible for the pharmaceutical activity of various influential drugs. ${ }^{26-29}$ In addition, pyridine derivatives represent numerous capabilities in diverse areas such as catalysis, coordination chemistry, polymer science, materials and surfaces engineering and agrochemistry. ${ }^{30-34}$ Therefore, pyridine and its derivatives (particularly 2-aminonicotinonitrile) have received extensive consideration and several efficacious methods have been developed by synthetic and medicinal chemists for their preparation. ${ }^{35-51}$

So, it is predicted that multicomponent synthesis ${ }^{52-56}$ of a single molecule bearing sulfonamide and pyridine centers simultaneously gives unique features to that structure inherited characteristics of both cores.

In addition to oxygen atom, which is responsible for anomeric interactions in various molecules, ${ }^{57-62}$ in non-oxygen heterocycles, other atoms such as nitrogen and sulfur play the role of donors in anomeric systems. For example, as depicted in \footnotetext{
Science and Technology, PO Box 447, Tengeru, Arusha, United Republic of Tanzania $\dagger$ Electronic supplementary information (ESI) available. See DOI: 10.1039/d0ra09400e

Department of Organic Chemistry, Faculty of Chemistry, Bu-Ali Sina University ${ }^{b}$ Institute of Nanotechnology and Water Sustainability (iNanoWS), College of Sc Engineering and Technology, University of South Africa, Johannesburg 1709, South 'UNESCO-UNISA Africa Chair in Nanosciences and Nanotechnology, College of Graduate Studies, Unive
Pretoria, South Africa

${ }^{d}$ Nanosciences African Network (NANOAFNET), iThemba LABS-National Research Foundation, 1 Old Faure Road, PO Box 722, Somerset West 7129, Western Cape, South Africa

${ }^{e}$ Department of Chemistry, Obafemi Awolowo University, Ile-Ife 220005, Nigeria ${ }^{f}$ School of Life Sciences and Bio-Engineering, The Nelson Mandela African Institution of
} 
<smiles>Nc1ccc(S(=O)(=O)Nc2ncccn2)cc1</smiles><smiles>Cc1ccc(-c2cc(C(F)(F)F)nn2-c2ccc(S(N)(=O)=O)cc2)cc1</smiles><smiles>NS(=O)(=O)Cc1noc2ccccc12</smiles><smiles>CCN[C@H]1C[C@H](C)S(=O)(=O)c2sc(S(N)(=O)=O)cc21</smiles><smiles>O=C(O)c1cc(N=Nc2ccc(S(=O)(=O)Nc3ccccn3)cc2)ccc1O</smiles><smiles>NCc1ccc(S(N)(=O)=O)cc1</smiles>

Mafenide

Scheme 1 Selected examples of drugs with sulfonamide structural core.

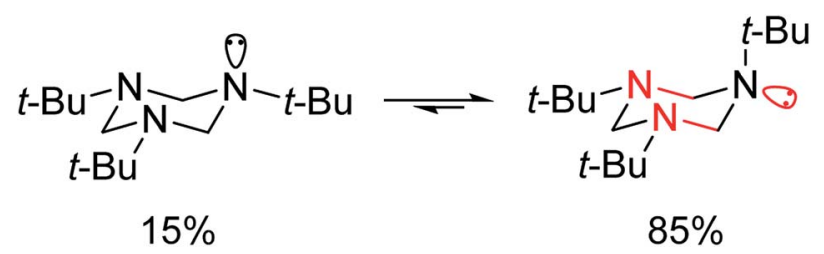

Scheme 2 Extension of anomeric effect to 1,3,5-tri-alkylhexahydro1,3,5-triazines.

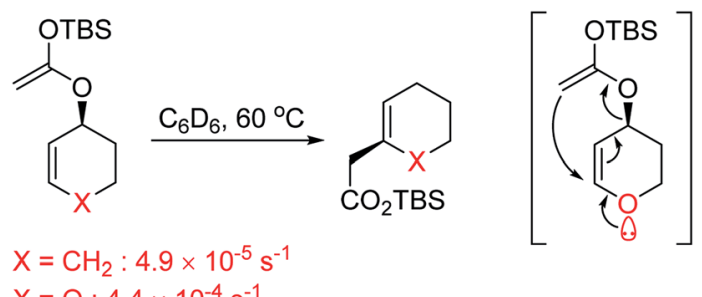

Scheme 3 The existence of the $\gamma$-allylic $O$-atom leads to acceleration of the Claisen rearrangement rate.

Scheme 2, due to stereoelectronic factors, in the case of 1,3,5-trialkylhexahydro-1,3,5-triazines, as a conformationally biased system even a bulky group such as tert-butyl occupied axial position (up to $85 \%)^{63,64}$ In this situation, the anomeric interaction of nitrogen lone pairs with vacant antibonding orbitals of carbon-nitrogen bonds $\left(\mathrm{n}_{\mathrm{N}} \rightarrow \sigma^{*}{ }_{\mathrm{C}-\mathrm{N}}\right)$ is possible.
In vinylogous anomeric effect, the anomeric interaction is transmitted from a donor through $\mathrm{C}=\mathrm{C}$ bonds to an acceptor. ${ }^{65}$ One of the striking examples for the vinylogous anomeric effect can be found in Claisen rearrangement. The existence of $\gamma$ allylic O-atom within the structure leads to acceleration of the reaction rate (Scheme 3). ${ }^{\mathbf{6 4 a}, \mathbf{6 6}}$ In these case, non-bonding electrons of $\gamma$-allylic $\mathrm{O}$-atom interact with antibonding of $\mathrm{C}-\mathrm{O}$ bond trough $\mathrm{C}=\mathrm{C}$ bond.

In this investigation, we continued our previous efforts for the synthesis of pyridine derivatives via anomeric or cooperative vinylogous anomeric-based oxidation mechanism ${ }^{67-73}$ and reported the preparation of a new series of pyridines with sulfonamide moiety in the presence of a novel quinoline-based dendrimer-like ionic liquid TQoxyTtriFA (Schemes 4 and 5).

\section{Results and discussion}

The systematic study of the anomeric effect in target biological candidated molecules allows the design of synthetic strategies based on anomerically driven stereoselective reactions, or highly biased equilibria among isomeric products. To the best of our knowledge, many biological processes involve oxidationreduction of substrates by $\mathrm{NAD}^{+} / \mathrm{NADH}$ and/or $\mathrm{NADP}^{+} / \mathrm{NADPH}$ systems, respectively. It is believed that the key feature of the oxidation mechanism in biological systems is the hydride transfer from carbon via anomeric-based oxidation. ${ }^{74-78}$ Thus, development of anomeric-based oxidation and/or
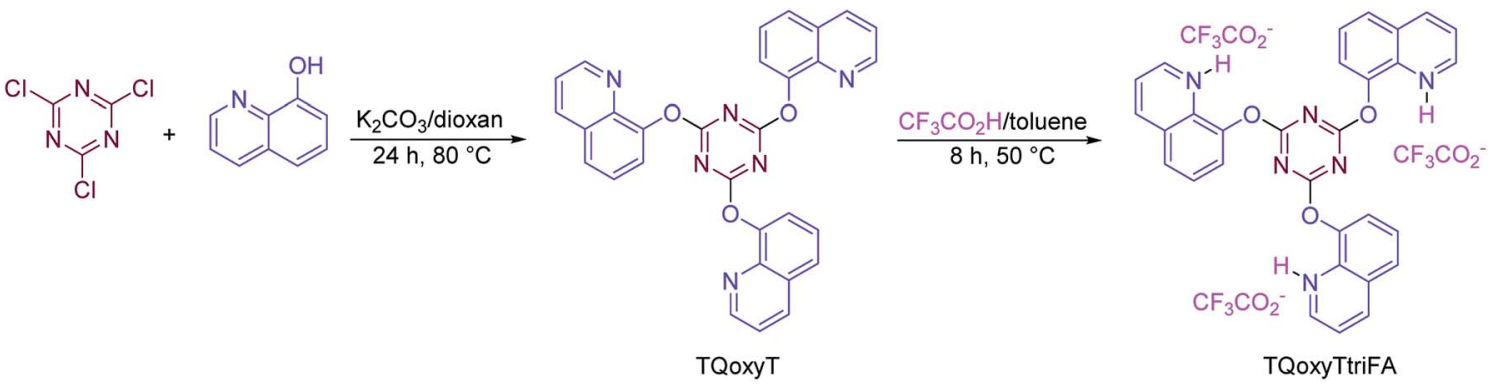

TQoxyTtriFA

Scheme 4 Synthetic route to TQoxyTtriFA as a novel quinoline-based dendrimer-like ionic liquid. 


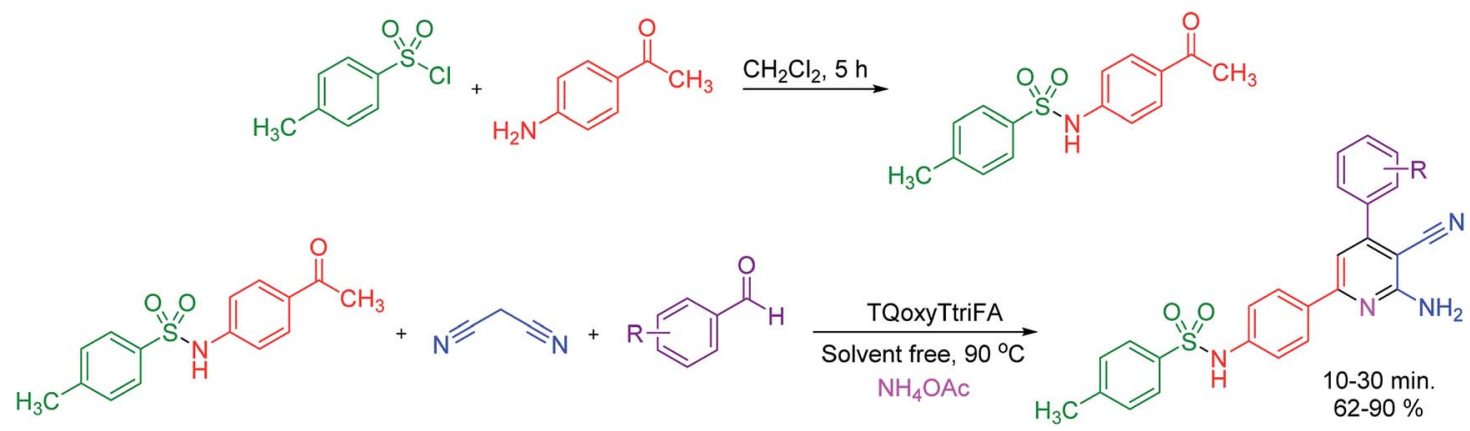

Scheme 5 Synthesis of new pyridines with sulfonamide moiety in the presence of TQoxyTtriFA.

aromatization mechanism leads to knowledge-based designing of biomimetic reactions in the future. With this aim, we decided to synthesize a range of new diversity of pyridines with sulfonamide moiety in the presence of a novel quinoline-based dendrimer-like ionic liquid.

The structure of the prepared novel quinoline-based dendrimer-like ionic liquid, namely TQoxyTtriFA was analyzed by several methods, which all endorsed each other. The more detailed data can be found in following.

The FT-IR spectrum of the catalyst verified the existence of characteristic functional groups including $\mathrm{C}=\mathrm{O}\left(1678 \mathrm{~cm}^{-1}\right)$ and acidic proton within the structure $\left(3446 \mathrm{~cm}^{-1}\right)$. Also, ${ }^{1} \mathrm{H}$ NMR spectrum is in agreement with the structure and confirms the symmetrical structure of the catalyst. This claim is in full agreement with the results obtained from ${ }^{13} \mathrm{C}$ NMR (for detail information, please see Spectral data section and ESI $\dagger$ ).

Fig. 1 depicts the output data of EDX analysis, which confirmed the elemental content of TQoxyTtriFA. The anticipated elements are carbon, nitrogen, oxygen and fluorine. Also, more verification was accomplished by applying the elemental mapping analysis (Fig. 2).

In a separate study, the thermal behavior of the prepared TQoxyTtriFA was investigated from $0{ }^{\circ} \mathrm{C}$ up to $600{ }^{\circ} \mathrm{C}$. The obtained TG and DTG plots are illustrated in Fig. 3. The weight loss occurred at around $150{ }^{\circ} \mathrm{C}$, indicating the high thermal stability of TQoxyTtriFA.

To prove the catalytic activity of TQoxyTtriFA as a novel quinoline-based dendrimer-like ionic liquid, we examined it for

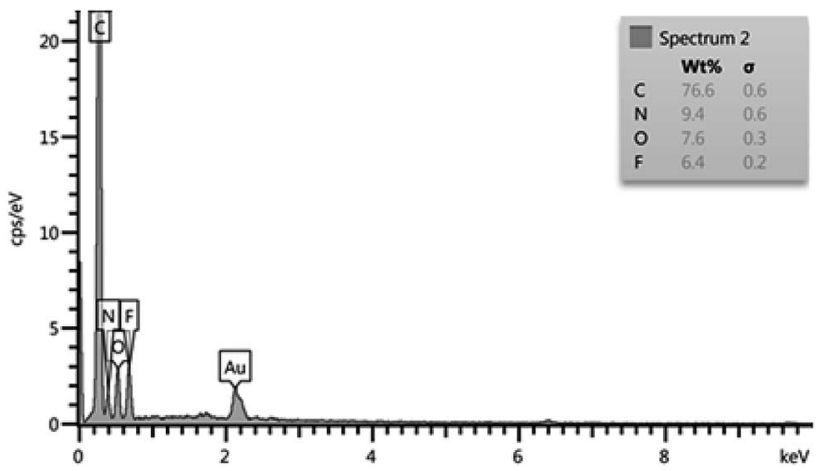

Fig. 1 Output data of EDX analysis of TQoxyTtriFA. the synthesis of a new pyridine series containing sulfonamide moiety. Our study began by examining the best reaction conditions by selecting a model reaction for the synthesis of molecule 1a. Our obtained data reveal that $90^{\circ} \mathrm{C}$ and solvent free conditions are the optimal reaction parameters. The data are collected in Table 1.

After determining the optimal conditions for the model reaction, we researched the productivity of the procedure for the synthesis of various pyridines containing sulfonamide moiety. The data, as collected in Table 2, show promising results. All new molecules were obtained in high yields at short reaction times via a catalytic one pot multicomponent reaction manner.

A plausible mechanism was suggested for the synthesis of 1a (Scheme 6). In this suggestion, Knoevenagel intermediate $\mathbf{A}$ (from the reaction of catalytic activated benzaldehyde and malononitrile) was treated with enamine intermediate $\mathbf{B}$ (from the reaction of in situ generated $\mathrm{NH}_{3}$ and $\mathrm{N}$-(4-acetylphenyl)-4methylbenzenesulfonamide). This catalytic process leads to the formation of intermediate $\mathbf{C}$. Then, in the presence of the new described catalyst, intermediate $\mathbf{C}$ is converted to intermediate $\mathbf{D}$, through a ring closer reaction. Afterwards, a tautomeric process leads to formation intermediate E. Finally, intermediate $\mathbf{E}$ undergoes a cooperative vinylogous anomeric-based oxidation (CVABO) mechanism for producing the target molecule 1a through unusual hydride transfer and releasing one molecular hydrogen $\left(\mathrm{H}_{2}\right)$. The achieved data from optimization of described reaction under argon and nitrogen atmospheres verified our suggestion for oxidation and aromatization of intermediate $\mathbf{E} .^{73,74}$

\section{Conclusion}

Here, the synthesis and characterization of a novel quinolinebased dendrimer-like IL was reported. Several skills were applied for studding the structure and formation of the novel IL. Also, the catalytic performance of titled IL was successfully employed for the facile synthesis of a new pyridine series containing sulfonamide moiety. Also, a plausible mechanism for the synthesis of target sulfonamide containing pyridines through a vinylogous anomeric-based oxidation mechanism was proposed. We think that the obtained results from this research will support the idea of rational designs, syntheses and applications of taskedspecific catalysts, susceptible biological candidate molecules and biomimetic reactions for the development of anomeric-based oxidation and/or aromatization mechanism. 

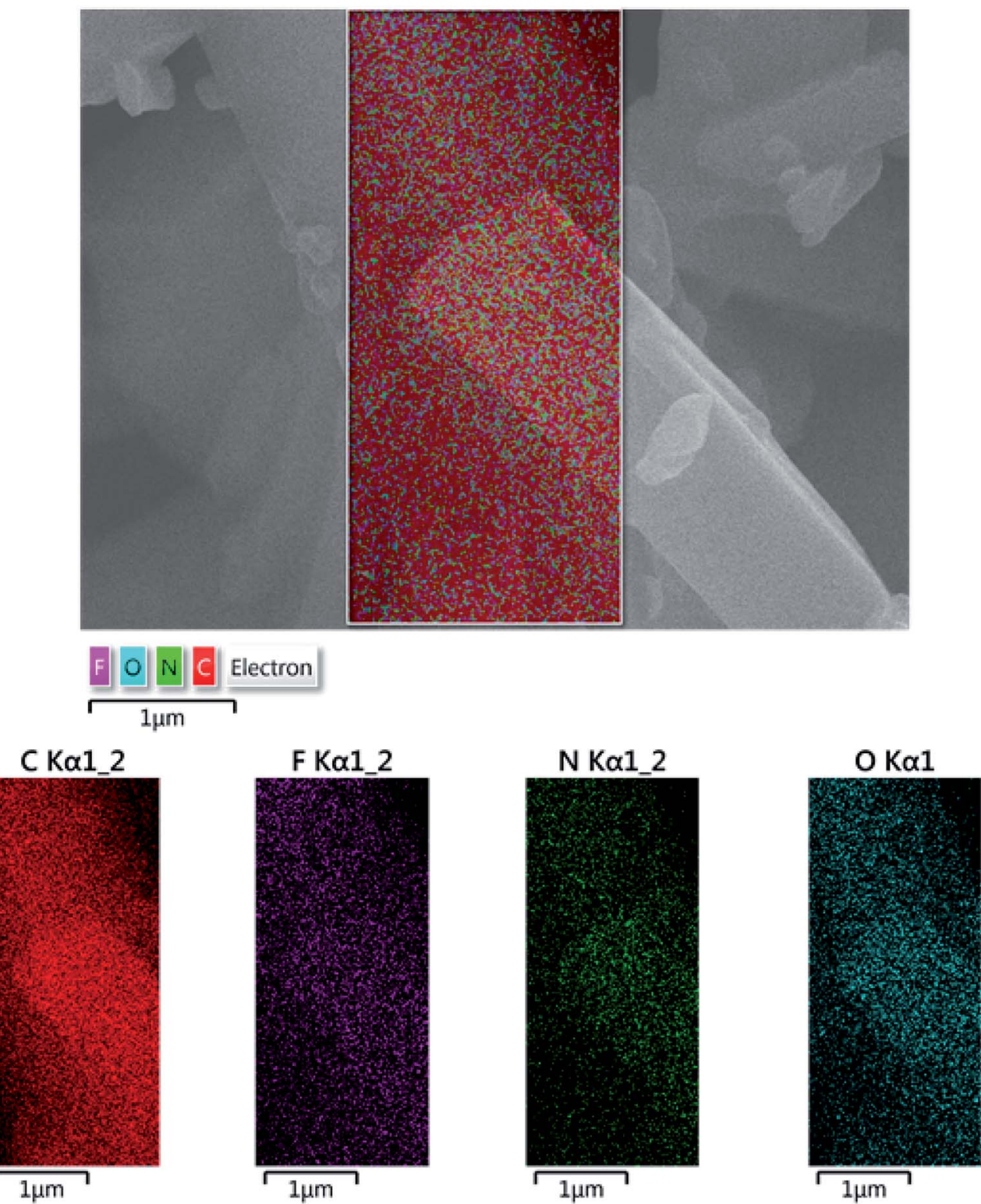

Fig. 2 Elemental mapping analysis of TQoxyTtriFA.

\section{Materials and methods}

Chemicals applied in this work for the synthesis of catalyst and products were supplied by Merck and Sigma-Aldrich chemical companies and used without further purification. Progress of the reactions was monitored by TLC using silica gel SIL G/UV 254 plates. Melting points were recorded on a Büchi B-545 apparatus in open capillary tubes. Fourier transform-infrared spectra (FT-IR) of the samples were recorded on a frontier FT-IR PerkinElmer with MID IR detector. The ${ }^{1} \mathrm{H}$ NMR spectra (301 MHz) and ${ }^{13} \mathrm{C}$ NMR (76 MHz) were recorded with a Bruker spectrometer or Varian Mercury 300 MHz Spectrometer (Varian Inv., Palo Alto, CA, USA) using $\mathrm{CDCl}_{3}$ or $\mathrm{DMSO}_{\mathrm{d} 6}$ as solvent. Thermal gravimetry analysis TG-DTG, were carried out on a STA 1500 from Rheometric Scientific at Day Petronic company. EDX and Elemental mapping analysis were performed using a SIGMA VP from Zeiss at Day Petronic company.
General procedure for the synthesis of TQoxyTtriFA as a novel quinoline-based dendrimer-like ionic liquid

At first, via the reaction of cyanuric chloride (1 eq.) and 8hydroxy quinoline ( 3 eq.), the related intermediate TQoxyT was prepared. ${ }^{79}$ Afterwards, the reaction of TQoxyT ( 1 eq.) with trifluoroacetic acid (3 eq.) in toluene as solvent at room temperature leads to formation of TQoxyTtriFA as a novel quinolinebased dendrimer-like ionic liquid (Scheme 4).

General procedure for the synthesis of $\mathrm{N}$-(4-acetylphenyl)-4methylbenzenesulfonamide

Similar to reported procedure, ${ }^{80} \quad N$-(4-Acetylphenyl)-4methylbenzenesulfonamide was prepared by the reaction of 4aminoacetophenone (10.0 mmol, $1.35 \mathrm{~g})$, 4-toluenesulfonyl chloride (10.0 mmol, $1.91 \mathrm{~g}$ ) and $2 \mathrm{~mol} \%$ of pyridine as catalyst in $\mathrm{CH}_{2} \mathrm{Cl}_{2}$ at room temperature for $5 \mathrm{~h}$. Afterwards, the solvent 


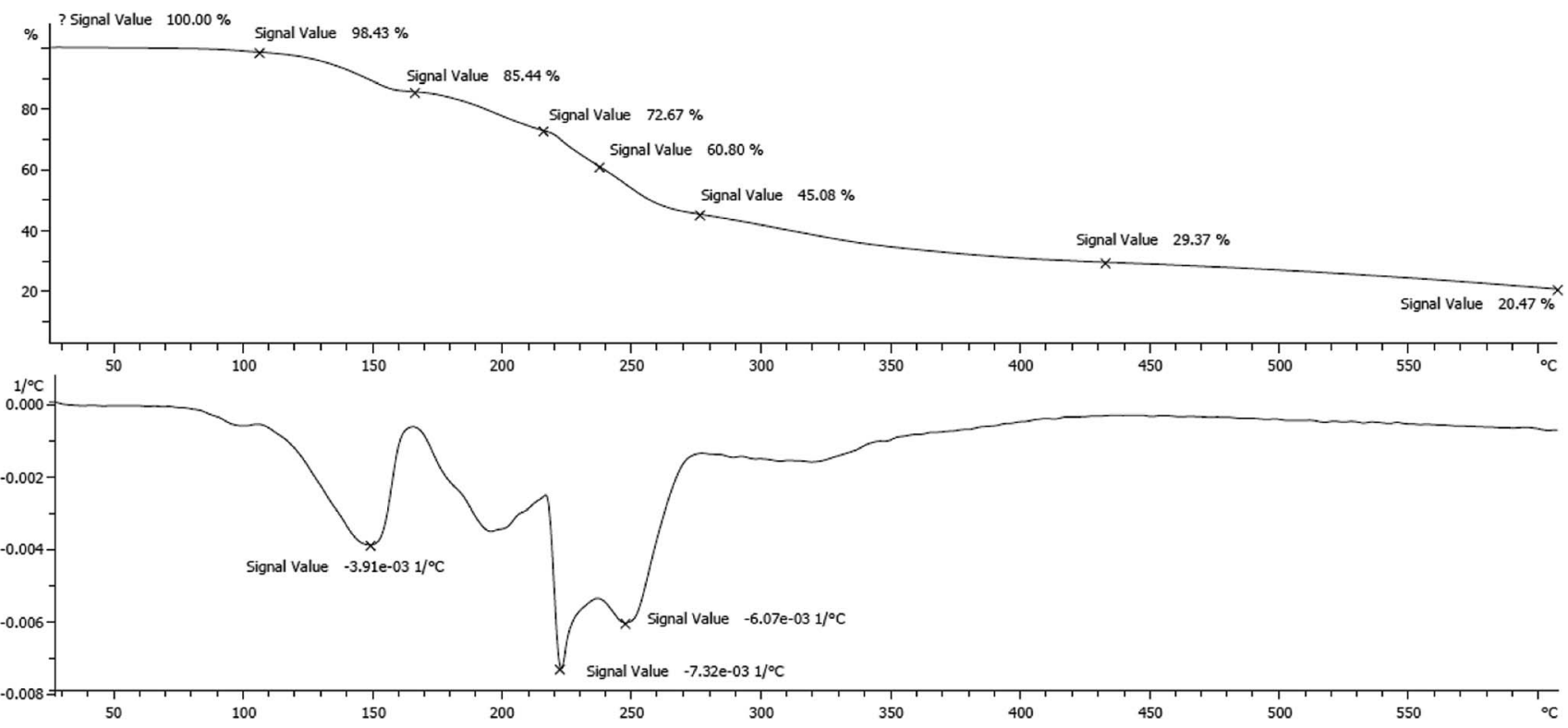

Fig. 3 TG and DTG plots of TQoxyTtriFA.

Table 1 Optimizing of the reaction conditions for the synthesis of $1 a^{a}$

\begin{tabular}{|c|c|c|c|c|c|}
\hline Entry & Solvent & Temperature $\left({ }^{\circ} \mathrm{C}\right)$ & Load of catalyst (mol\%) & Time (min) & Yield $^{b}(\%)$ \\
\hline 1 & - & 100 & 2 & 15 & 84 \\
\hline 2 & - & 90 & 5 & 15 & 82 \\
\hline 4 & & 90 & 1 & 20 & 71 \\
\hline 5 & - & 90 & - & 15 & Trace \\
\hline 6 & - & 90 & - & 120 & 52 \\
\hline 9 & - & 60 & 2 & 15 & 20 \\
\hline 10 & - & 60 & 2 & 60 & 55 \\
\hline 11 & $\mathrm{H}_{2} \mathrm{O}$ & Reflux & 2 & 120 & - \\
\hline 12 & EtOH & Reflux & 2 & 120 & 40 \\
\hline 13 & $n$-Hexane & Reflux & 2 & 120 & - \\
\hline 14 & EtOAc & Reflux & 2 & 120 & - \\
\hline
\end{tabular}

${ }^{a}$ Reaction condition: benzaldehyde ( $\left.1 \mathrm{mmol}, 0.106 \mathrm{~g}\right)$, malononitrile ( $\left.1 \mathrm{mmol}, 0.066 \mathrm{~g}\right), \mathrm{N}$-(4-acetylphenyl)-4-methylbenzenesulfonamide ( 1 mmol, $0.289 \mathrm{~g}$ ) and ammonium acetate $(1 \mathrm{mmol}, 0.077 \mathrm{~g}) .{ }^{b}$ Isolated yields. ${ }^{c}$ The achieved data from optimization of described reaction under air, argon and nitrogen atmospheres are similar.

was removed and the obtained solid was filtered and was thoroughly washed with water and air dried to afford the pure product (yields up to $88 \%$ ).

\section{General procedure for the synthesis of new pyridines with sulfonamide moiety in the presence of TQoxyTtriFA}

The general protocol for the synthesis of sulfonamide containing pyridines are as follows. To a mixture of aryl aldehyde (1.0 $\mathrm{mmol}$ ), malononitrile (1.0 mmol, $0.066 \mathrm{~g}), \mathrm{N}$-(4-acetylphenyl)-4methylbenzenesulfonamide $(1.0 \mathrm{mmol}, 0.289 \mathrm{~g})$ and $\mathrm{NH}_{4} \mathrm{OAc}$ (1.0 mmol, $0.077 \mathrm{~g}$ ), 2 mol\% of TQoxyTtriFA was added as catalyst. Then, the mixture was stirred and heated at $90{ }^{\circ} \mathrm{C}$ under solvent free conditions for appropriate times as reported in Table 2 . The reaction progress and the purity of the molecules were checked out by TLC ( $n$-hexane/ethylacetate $(1: 1)$. Eventually, the products were purified by using prepared silica plates using a mixture $n$-hexane/ethylacetate $(1: 1)$.

\section{Spectral data}

TQoxyTtriFA (quinoline-based dendrimer-like ionic liquid). $\mathrm{MP}=94-96{ }^{\circ} \mathrm{C}$, FT-IR (KBr, $\left.\nu, \mathrm{cm}^{-1}\right): 3446,3075,1677,1606$, 1268. ${ }^{1} \mathrm{H}$ NMR (301 MHz, DMSO) $\delta_{\mathrm{ppm}} 9.70$ (bs, $\left.1 \mathrm{H}, \mathrm{NH}\right), 8.85$ (dd, $3 \mathrm{H}, J=4.3,1.7 \mathrm{~Hz}$, aromatic), $8.42(\mathrm{dd}, 3 \mathrm{H}, J=8.4,1.7 \mathrm{~Hz}$, aromatic), 7.85 (dd, 3H, $J=7.4,2.2 \mathrm{~Hz}$, aromatic), $7.62-7.49(\mathrm{~m}$, 
Table 2 Catalytic synthesis of a new library of pyridines with sulfonamide moiety ${ }^{a}$

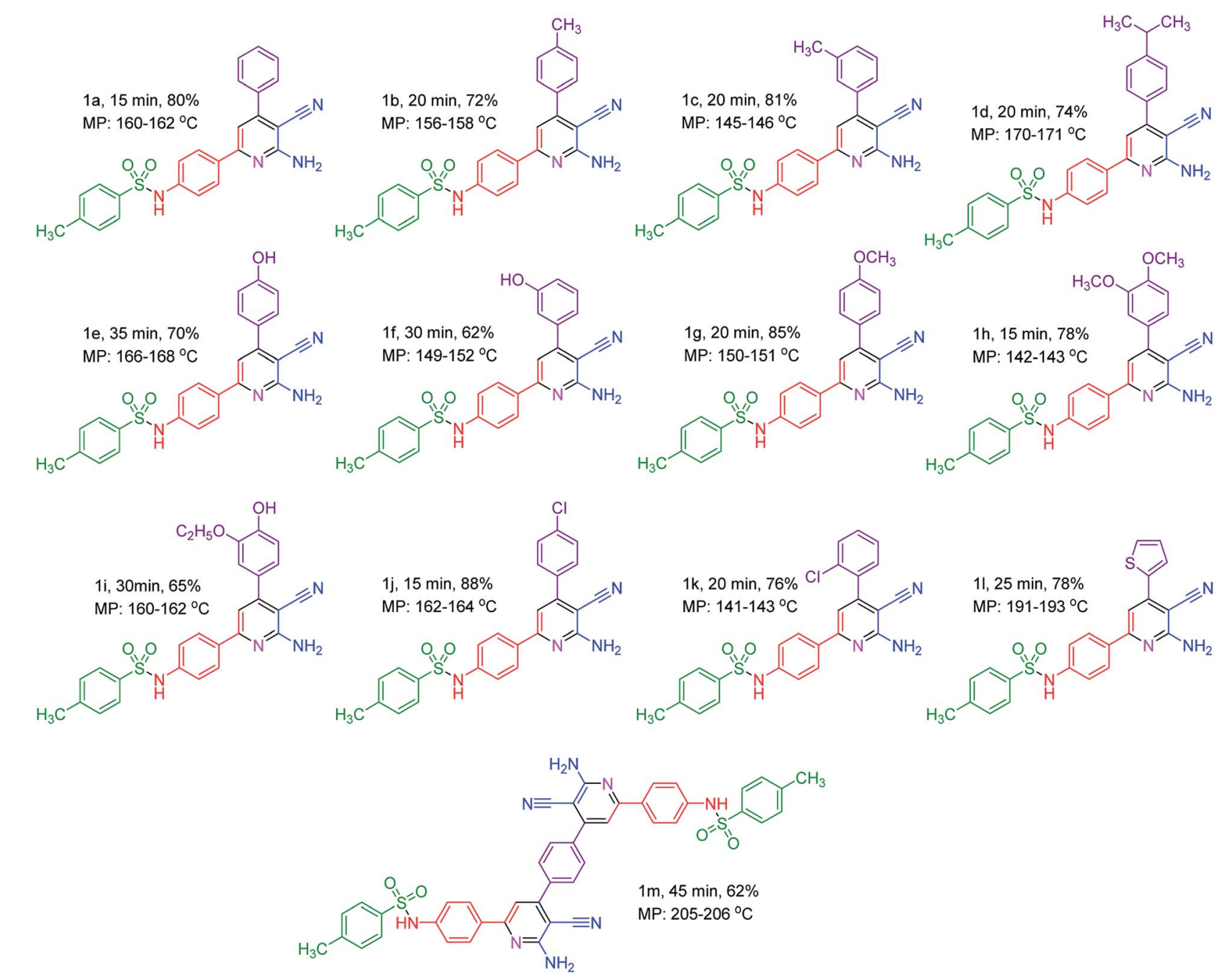

${ }^{a}$ Reaction conditions: aryl aldehyde (1.0 mmol), malononitrile (1.0 mmol, $\left.0.066 \mathrm{~g}\right), N$-(4-acetylphenyl)-4-methylbenzenesulfonamide (1.0 mmol, $0.289 \mathrm{~g})$ and ammonium acetate $(1.0 \mathrm{mmol}, 0.077 \mathrm{~g})$, solvent free, $90^{\circ} \mathrm{C}$, catalyst $=2 \mathrm{~mol} \%$. Reported yields are referred to isolated yields.

9H, aromatic). ${ }^{13} \mathrm{C}$ NMR (76 MHz, DMSO) $\delta 174.1,159.6,159.1$, $158.6,158.2$, 150.7, 147.2, 139.8, 137.2, 129.5, 126.9, 126.8, 122.7, 121.8, 117.5, 113.7 .

$\mathrm{N}$-(4-Acetylphenyl)-4-methylbenzenesulfonamide. $\mathrm{MP}=$ 196-198 ${ }^{\circ} \mathrm{C}$, FT-IR (KBr, $\left.\nu, \mathrm{cm}^{-1}\right): 3221,3000,1671,1600,1342$, 1281, 1158, 912. ${ }^{1} \mathrm{H}$ NMR (301 MHz, DMSO) $\delta_{\mathrm{ppm}} 10.18(\mathrm{~s}, 1 \mathrm{H}$, $\mathrm{NH}), 7.21$ (d, $2 \mathrm{H}, J=9 \mathrm{~Hz}$, aromatic), 7.11 (d, $2 \mathrm{H}, J=9 \mathrm{~Hz}$, aromatic), 6.74 (d, $2 \mathrm{H}, J=6 \mathrm{~Hz}$, aromatic), $6.60(\mathrm{~d}, 2 \mathrm{H}, J=9 \mathrm{~Hz}$, aromatic), $1.84\left(\mathrm{~s}, 3 \mathrm{H}, \mathrm{CH}_{3}\right), 1.70\left(\mathrm{~s}, 3 \mathrm{H}, \mathrm{CH}_{3}\right) .{ }^{13} \mathrm{C} \mathrm{NMR}(76$ MHz, DMSO) $\delta_{\text {ppm }} 197.0,144.3,142.9,137.0,132.5,130.4,130.4$, 127.3, 118.4, 26.9, 21.5.

N-(4-(6-Amino-5-cyano-4-phenylpyridin-2-yl)phenyl)-4methylbenzenesulfonamide (1a). $\mathrm{MP}=160-162{ }^{\circ} \mathrm{C}$, FT-IR $(\mathrm{KBr}$, $\left.\nu, \mathrm{cm}^{-1}\right)$ : 3548, 3407, 3284, 2924, 2215, 1612, 1573, 1337, 1158, 921. ${ }^{1} \mathrm{H}$ NMR (301 MHz, $\left.\mathrm{CDCl}_{3}\right) \delta_{\mathrm{ppm}} 8.14-8.09(\mathrm{~m}, 2 \mathrm{H}$, aromatic), 7.96-7.91 (m, 2H, aromatic), 7.86-7.80 (m, $2 \mathrm{H}$, aromatic), 7.76-7.71 (m, 3H, aromatic), 7.49-7.32 (m, 6H, aromatic and $\mathrm{NH}$ ), 5.60 (bs, $\left.2 \mathrm{H}, \mathrm{NH}_{2}\right), 2.61\left(\mathrm{~s}, 3 \mathrm{H}, \mathrm{CH}_{3}\right) \cdot{ }^{13} \mathrm{C}$ NMR (76 MHz, $\mathrm{CDCl}_{3}$ ) $\delta_{\mathrm{ppm}} 160.3,158.8,155.4,144.4,138.6$, $137.0,136.1,134.5,130.1,130.0,129.1,128.6$, 128.3, 127.4, 120.8, 117.3, 111.0, 88.3, 21.7. TOF-HRMS (EI): $m / z(\mathrm{M})^{+}+1$ calcd for $\mathrm{C}_{25} \mathrm{H}_{20} \mathrm{~N}_{4} \mathrm{O}_{2} \mathrm{~S}$ 441.1307; found 441.1199.

N-(4-(6-Amino-5-cyano-4-(p-tolyl)pyridin-2-yl)phenyl)-4methylbenzenesulfonamide (1b). $\mathrm{MP}=156-158^{\circ} \mathrm{C}$, FT-IR $(\mathrm{KBr}$, $\left.\nu, \mathrm{cm}^{-1}\right)$ : 3521, 3396, 3276, 2929, 2217, 1610, 1577, 1339, 1159, 821. ${ }^{1} \mathrm{H}$ NMR $\left(301 \mathrm{MHz}, \mathrm{CDCl}_{3}\right) \delta_{\mathrm{ppm}} 8.01(\mathrm{~d}, 2 \mathrm{H}, J=9 \mathrm{~Hz}$, aromatic), $7.86(\mathrm{~d}, 2 \mathrm{H}, J=9 \mathrm{~Hz}$, aromatic), $7.66(\mathrm{~d}, 2 \mathrm{H}, J=9 \mathrm{~Hz}$, aromatic), 7.47 (d, $2 \mathrm{H}, J=9 \mathrm{~Hz}$, aromatic), $7.38(\mathrm{~d}, 2 \mathrm{H}, J=9 \mathrm{~Hz}$, aromatic), 7.32-7.29 (m, 3H, aromatic), $7.24(\mathrm{~s}, 1 \mathrm{H}, \mathrm{NH}), 5.51$ (bs, $\left.2 \mathrm{H}, \mathrm{NH}_{2}\right), 2.58\left(\mathrm{~s}, 3 \mathrm{H}, \mathrm{CH}_{3}\right), 2.51\left(\mathrm{~s}, 3 \mathrm{H}, \mathrm{CH}_{3}\right) .{ }^{13} \mathrm{C} \mathrm{NMR}(76$ $\left.\mathrm{MHz}, \mathrm{CDCl}_{3}\right) \delta_{\text {ppm }} 160.4,158.7,155.4,144.3,140.3,138.6,136.1$, $134.6,134.1$, 129.9, 129.8, 128.6, 128.2, 127.4, 120.7, 117.4, $110.9,88.2,21.7,21.5$. 


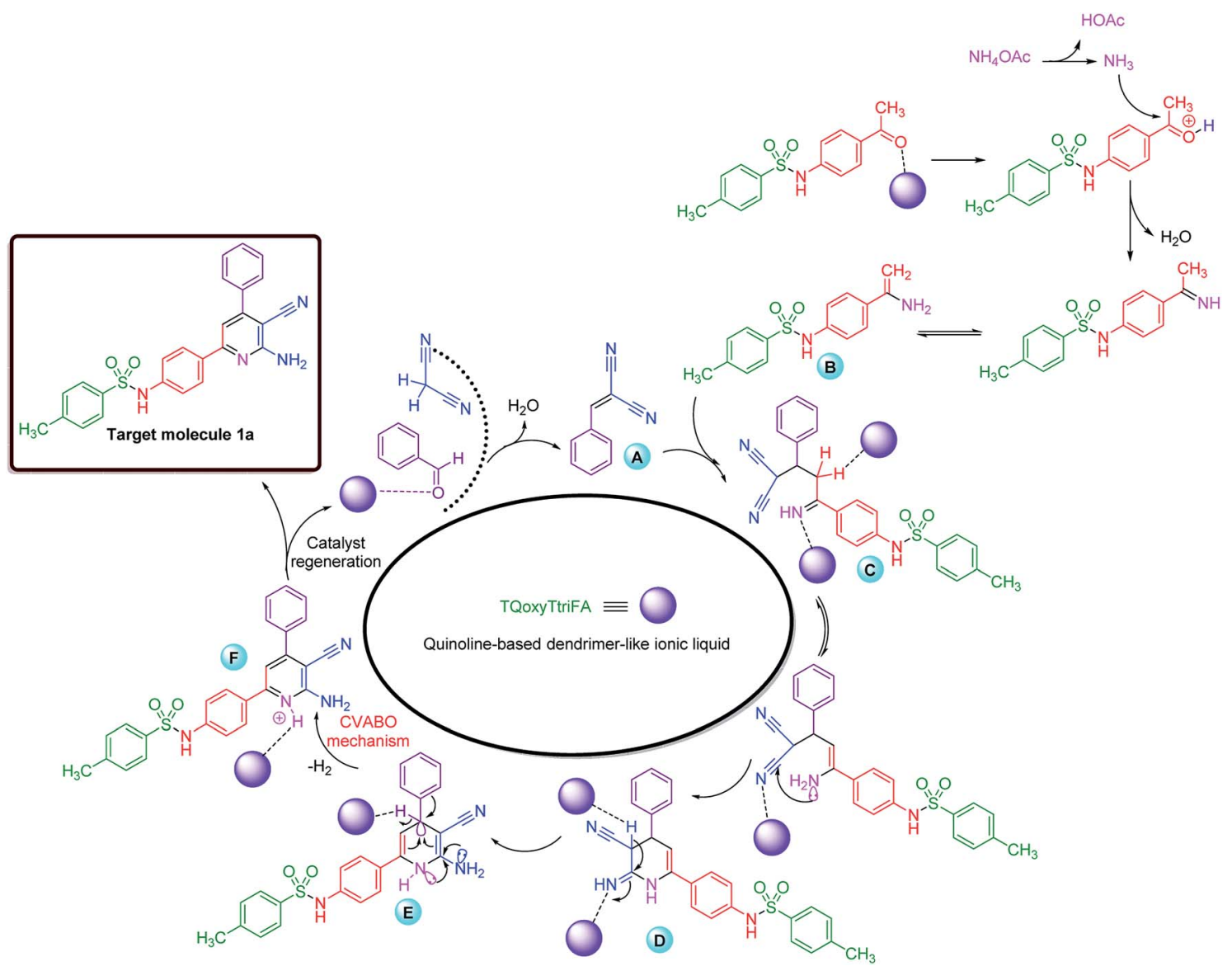

Scheme 6 A catalytic plausible cooperative vinylogous anomeric-based oxidation (CVABO) mechanism for the synthesis of 1a.

$N$-(4-(6-Amino-5-cyano-4-(m-tolyl)pyridin-2-yl)phenyl)-4-

methylbenzenesulfonamide (1c). MP $=145-146^{\circ} \mathrm{C}$, FT-IR $(\mathrm{KBr}$, $\left.\nu, \mathrm{cm}^{-1}\right)$ : 3520, 3389, 3273, 2982, 2218, 1611, 1553, 1336, 1158. ${ }^{1} \mathrm{H}$ NMR (301 MHz, $\left.\mathrm{CDCl}_{3}\right) \delta_{\mathrm{ppm}} 8.02$ (d, 2H, $J=9 \mathrm{~Hz}$, aromatic), 7.83 (d, $2 \mathrm{H}, J=9 \mathrm{~Hz}$, aromatic), 7.52-7.53 (m, 3H, aromatic), 7.45-7.44 (m, 1H, aromatic), 7.38-7.34 (m, 3H, aromatic), 7.29 (d, $2 \mathrm{H}, J=9 \mathrm{~Hz}$, aromatic), $7.23(\mathrm{~s}, 1 \mathrm{H}, \mathrm{NH}), 5.45$ (bs, $2 \mathrm{H}, \mathrm{NH}_{2}$ ), 2.57 (s, 3H, $\mathrm{CH}_{3}$ ), 2.50 (s, 3H, $\mathrm{CH}_{3}$ ). ${ }^{13} \mathrm{C} \mathrm{NMR}\left(76 \mathrm{MHz}, \mathrm{CDCl}_{3}\right.$ ) $\delta_{\text {ppm }} 160.2,158.6,155.4,144.3,138.5,136.4,134.5,131.1,130.7$, 129.8, 128.9, 128.8, 128.5, 127.3, 125.3, 120.7, 117.2, 110.9, 88.3, 21.6, 21.5. TOF-HRMS (EI): $m / z(\mathrm{M})^{+}+1$ calcd for $\mathrm{C}_{26} \mathrm{H}_{22} \mathrm{~N}_{4} \mathrm{O}_{2} \mathrm{~S}$ 455.1463; found 455.1349 .

$\mathrm{N}$-(4-(6-Amino-5-cyano-4-(4-isopropylphenyl)pyridin-2-yl) phenyl)-4-methylbenzenesulfonamide (1d). $\mathrm{MP}=170-171{ }^{\circ} \mathrm{C}$, FT-IR (KBr, $\left.\nu, \mathrm{cm}^{-1}\right)$ : 358, 3396, 3252, 2923, 2216, 1615, 1464, 1340, 1160, 818. ${ }^{1} \mathrm{H} \mathrm{NMR}\left(301 \mathrm{MHz}, \mathrm{CDCl}_{3}\right) \delta_{\mathrm{ppm}} 8.08(\mathrm{~d}, 2 \mathrm{H}, J=$ $9 \mathrm{~Hz}$, aromatic), 7.91 (d, $2 \mathrm{H}, J=9 \mathrm{~Hz}$, aromatic), 7.75 (d, 2H, $=$ $6 \mathrm{~Hz}$, aromatic), 7.57 (d, 2H, $J=9 \mathrm{~Hz}$, aromatic), 7.43 (d, 2H, $J=$ $9 \mathrm{~Hz}$, aromatic), 7.37-7.34 (m, 3H, aromatic), $7.31(\mathrm{~s}, 1 \mathrm{H}, \mathrm{NH})$, 5.54 (bs, $2 \mathrm{H}, \mathrm{NH}_{2}$ ), $3.18(\mathrm{~s}, 1 \mathrm{H}, J=6 \mathrm{~Hz}, \mathrm{CH}), 2.57\left(\mathrm{~s}, 3 \mathrm{H}, \mathrm{CH}_{3}\right)$, $1.50\left(\mathrm{~d}, 6 \mathrm{H}, J=6 \mathrm{~Hz}, \mathrm{CH}_{3}\right) \cdot{ }^{13} \mathrm{C} \mathrm{NMR}\left(76 \mathrm{MHz}, \mathrm{CDCl}_{3}\right) \delta_{\mathrm{ppm}}$ $160.4,158.7,155.4,151.2$, 144.4, 138.6, 136.09, 134.7, 134.4, 130.0, 128.6, 128.3, 128.2, 127.4, 127.3, 120.8, 117.5, 111.0, 88.2, 34.2, 24.0, 21.7. TOF-HRMS (EI): $m / z(\mathrm{M})^{+}+1$ calcd for $\mathrm{C}_{28} \mathrm{H}_{26} \mathrm{~N}_{4} \mathrm{O}_{2} \mathrm{~S}$ 483.1776; found 483.1651.
$\mathrm{N}$-(4-(6-Amino-5-cyano-4-(4-hydroxyphenyl)pyridin-2-yl) phenyl)-4-methylbenzenesulfonamide (1e). MP $=166-168{ }^{\circ} \mathrm{C}$, ${ }^{1} \mathrm{H}$ NMR (301 MHz, DMSO) $\delta_{\text {ppm }} 7.65-7.57$ (m, 2H, aromatic), 7.39-7.32 (m, 2H, aromatic), 7.20-7.14 (m, 2H, aromatic), 7.036.96 (m, 3H, aromatic), 6.85-6.75 (m, 4H, aromatic), 6.60-6.54 $(\mathrm{m}, 2 \mathrm{H}$, aromatic and $\mathrm{NH}), 6.46\left(\mathrm{bs}, 2 \mathrm{H}, \mathrm{NH}_{2}\right), 7.21-7.15(\mathrm{~m}, 3 \mathrm{H}$, $\left.\mathrm{CH}_{3}\right) .{ }^{13} \mathrm{C}$ NMR (76 MHz, DMSO) $\delta_{\mathrm{ppm}} 161.6,159.6,158.5,155.2$, $143.5,138.2$, 137.3, 132.3, 130.5, 130.3, 128.8, 128.2, 127.4, $119.8,118.3,116.2,108.9,86.1,21.6$. TOF-HRMS (EI): $\mathrm{m} / z(\mathbf{M})^{+}+$ 1 calcd for $\mathrm{C}_{25} \mathrm{H}_{20} \mathrm{~N}_{4} \mathrm{O}_{3} \mathrm{~S}$ 457.1256; found 457.1143.

$\mathrm{N}$-(4-(6-Amino-5-cyano-4-(3-hydroxyphenyl)pyridin-2-yl) phenyl)-4-methylbenzenesulfonamide (1f). $\mathrm{MP}=149-152{ }^{\circ} \mathrm{C}$, ${ }^{1} \mathrm{H}$ NMR (301 MHz, DMSO) $\delta_{\text {ppm }} 8.18-8.14$ (m, 2H, aromatic), 7.92-7.87 (m, 2H, aromatic), 7.56-7.52 (m, 4H, aromatic), 7.387.29 (m, 4H, aromatic), 7.23-7.21 (m, 2H, aromatic), 7.13-7.07 $\left(\mathrm{m}, 3 \mathrm{H}, \mathrm{NH}\right.$ and $\left.\mathrm{NH}_{2}\right), 2.74-2.68\left(\mathrm{~m}, 3 \mathrm{H}, \mathrm{CH}_{3}\right) .{ }^{13} \mathrm{C} \mathrm{NMR}(76$ MHz, DMSO) $\delta_{\mathrm{ppm}} 161.4,158.7,158.2,155.4,143.4,139.1,138.5$, $132.1,130.5,130.2,128.8,127.4,119.8,119.5,117.8,117.1$, 115.68, 109.1, 86.4, 21.6.

$\mathrm{N}$-(4-(6-Amino-5-cyano-4-(4-methoxyphenyl)pyridin-2-yl) phenyl)-4-methylbenzenesulfonamide (1g). MP $=150-151{ }^{\circ} \mathrm{C}$, FT-IR (KBr, $\nu, \mathrm{cm}^{-1}$ ): 3525, 3396, 3259, 2924, 2213, 1609, 1513, 1379, 1157, 829. ${ }^{1} \mathrm{H}$ NMR (301 MHz, $\left.\mathrm{CDCl}_{3}\right) \delta_{\mathrm{ppm}} 7.94(\mathrm{~d}, 2 \mathrm{H}, J=$ $9 \mathrm{~Hz}$, aromatic), 7.79 (d, $2 \mathrm{H}, J=9 \mathrm{~Hz}$, aromatic), $7.66(\mathrm{~d}, 2 \mathrm{H}, J=$ $9 \mathrm{~Hz}$, aromatic), 7.37-7.29 (m, 3H, aromatic), 7.26-7.21 (m, $2 \mathrm{H}$, 
aromatic), $7.15(\mathrm{~s}, 1 \mathrm{H}, \mathrm{NH}), 7.10(\mathrm{~d}, 2 \mathrm{H}, J=6 \mathrm{~Hz}$, aromatic), 5.42 (bs, 2H, NH $\mathrm{NH}_{2}$ ), $3.95\left(\mathrm{~s}, 3 \mathrm{H}, \mathrm{OCH}_{3}\right), 2.44\left(\mathrm{~s}, 3 \mathrm{H}, \mathrm{CH}_{3}\right) \cdot{ }^{13} \mathrm{C} \mathrm{NMR}(76$ $\left.\mathrm{MHz} \mathrm{CDCl}_{3}\right) \delta_{\text {ppm }} 161.1,160.4,158.6,154.9,144.2,138.5,136.1$, $134.6,129.9,129.7,129.2,128.5,127.4,120.7,117.5,114.5$, 110.7, 88.0, 55.5, 21.6. TOF-HRMS (EI): $m / z(\mathrm{M})^{+}+1$ calcd for $\mathrm{C}_{26} \mathrm{H}_{22} \mathrm{~N}_{4} \mathrm{O}_{3} \mathrm{~S}$ 471.1413; found 471.1299.

$\mathrm{N}$-(4-(6-Amino-5-cyano-4-(3,4-dimethoxyphenyl)pyridin-2-yl) phenyl)-4-methylbenzenesulfonamide (1h). $\mathrm{MP}=142-143{ }^{\circ} \mathrm{C}$, FT-IR (KBr, $\left.\nu, \mathrm{cm}^{-1}\right): 3525,3390,3262,2923,2214,1614,1514$, 1337, 1260, 1159, 816. ${ }^{1} \mathrm{H}$ NMR (301 MHz, $\left.\mathrm{CDCl}_{3}\right) \delta_{\mathrm{ppm}} 7.94(\mathrm{~d}$, $2 \mathrm{H}, J=9 \mathrm{~Hz}$, aromatic), $7.74(\mathrm{~d}, 2 \mathrm{H}, J=9 \mathrm{~Hz}$, aromatic), 7.317.19 (m, 7H, aromatic), 7.15 (s, $1 \mathrm{H}, \mathrm{NH}), 7.04$ (d, $2 \mathrm{H}, J=9 \mathrm{~Hz}$, aromatic), 5.37 (bs, $\left.2 \mathrm{H}, \mathrm{NH}_{2}\right), 4.0\left(\mathrm{t}, 6 \mathrm{H}, J=1.5 \mathrm{~Hz}, \mathrm{OCH}_{3}\right), 2.42$ $\left(\mathrm{s}, 3 \mathrm{H}, \mathrm{CH}_{3}\right) .{ }^{13} \mathrm{C} \mathrm{NMR}\left(76 \mathrm{MHz}, \mathrm{CDCl}_{3}\right) \delta_{\mathrm{ppm}} 160.4,158.6,155.0$, $150.7,149.2$, 144.3, 138.5, 134.7, 129.9, 129.4, 128.6, 127.4, $121.2,120.7,117.9,111.4,111.3,110.7,56.26$, 56.2, 21.7. TOFHRMS (EI): $m / z(M)^{+}+1$ calcd for $\mathrm{C}_{27} \mathrm{H}_{24} \mathrm{~N}_{4} \mathrm{O}_{4} \mathrm{~S}$ 501.1518; found 501.1393 .

N-(4-(6-Amino-5-cyano-4-(3-ethoxy-4-hydroxyphenyl)pyridin2-yl)phenyl)-4-methylbenzenesulfonamide (1i). $\mathrm{MP}=160$ $162{ }^{\circ} \mathrm{C}$, FT-IR (KBr, $\left.\nu, \mathrm{cm}^{-1}\right): 3509,3392,3264,2924,2212,1609$, 1513, 1338, 1267, 1157, 821. ${ }^{1} \mathrm{H}$ NMR (301 MHz, $\left.\mathrm{CDCl}_{3}\right) \delta_{\mathrm{ppm}}$ 7.85 (d, $2 \mathrm{H}, J=9 \mathrm{~Hz}$, aromatic), 7.71 (d, $2 \mathrm{H}, J=9 \mathrm{~Hz}$, aromatic), 7.22 (d, $2 \mathrm{H}, J=9 \mathrm{~Hz}$, aromatic), 7.16-7.09 (m, 5H, aromatic), 7.07 (s, 1H, NH), 7.04-7.01 (d, 2H, $J=9 \mathrm{~Hz}$, aromatic), 5.36 (bs, $2 \mathrm{H}, \mathrm{NH}_{2}$ ), 4.18 (q, $\left.2 \mathrm{H}, J=9 \mathrm{~Hz}, \mathrm{CH}_{2}\right), 2.35\left(\mathrm{~s}, 3 \mathrm{H}, \mathrm{CH}_{3}\right), 1.47$ (t, $\left.3 \mathrm{H}, J=6 \mathrm{~Hz}, \mathrm{CH}_{3}\right) .{ }^{13} \mathrm{C} \mathrm{NMR}\left(76 \mathrm{MHz}, \mathrm{CDCl}_{3}\right) \delta_{\mathrm{ppm}} 160.5,158.7$, $155.1,147.6,146.0,144.3,138.6,136.2,134.5$, 129.9, 128.8, 128.5, 127.4, 121.7, 120.7, 117.6, 115.0, 111.7, 110.7, 87.9, 65.0, 21.6, 14.9. TOF-HRMS (EI): $m / z(M)^{+}+1$ calcd for $\mathrm{C}_{27} \mathrm{H}_{24} \mathrm{~N}_{4} \mathrm{O}_{4} \mathrm{~S}$ 501.1518; found 501.1386.

$\mathrm{N}$-(4-(6-Amino-4-(4-chlorophenyl)-5-cyanopyridin-2-yl) phenyl)-4-methylbenzenesulfonamide (1j). $\mathrm{MP}=182-184{ }^{\circ} \mathrm{C}$, ${ }^{1} \mathrm{H}$ NMR (301 MHz, DMSO) $\delta_{\mathrm{ppm}} 7.93$ (d, 2H, $J=3 \mathrm{~Hz}$, aromatic), 7.68-7.59 (m, 6H, aromatic), 7.30 (d, $2 \mathrm{H}, J=6 \mathrm{~Hz}$, aromatic), $7.21(\mathrm{t}, 1 \mathrm{H}, J=6 \mathrm{~Hz}$, aromatic), 7.14-7.09 (m, 3H, aromatic and $\mathrm{NH}), 6.94$ (bs, $\left.2 \mathrm{H}, \mathrm{NH}_{2}\right), 2.31\left(\mathrm{~s}, 3 \mathrm{H}, \mathrm{CH}_{3}\right) .{ }^{13} \mathrm{C} \mathrm{NMR}(76 \mathrm{MHz}$, DMSO) $\delta_{\mathrm{ppm}} 161.3,159.0,153.8,143.0,136.5,135.0,130.8$, 130.2 , 130.0, 129.6, 129.3, 128.7, 127.2, 119.8, 119.0, 117.6, 109.0, 86.1, 21.5.

$\mathrm{N}$-(4-(6-Amino-4-(2-chlorophenyl)-5-cyanopyridin-2-yl) phenyl)-4-methylbenzenesulfonamide (1k). MP $=141-143{ }^{\circ} \mathrm{C}$, FT-IR (KBr, $\nu, \mathrm{cm}^{-1}$ ): 3441, 3342, 3248, 2928, 2216, 1639, 1556, 1334, 1161, 759. ${ }^{1} \mathrm{H} \mathrm{NMR}\left(301 \mathrm{MHz}, \mathrm{CDCl}_{3}\right) \delta_{\mathrm{ppm}} 8.04(\mathrm{~d}, 2 \mathrm{H}, J=$ $9 \mathrm{~Hz}$, aromatic), 7.90 (d, $2 \mathrm{H}, J=9 \mathrm{~Hz}$, aromatic), $7.71(\mathrm{~d}, 1 \mathrm{H}, J=$ $6 \mathrm{~Hz}$, aromatic), 7.59-7.51 (m, 3H, aromatic), 7.44-7.32 (m, 5H, aromatic), 7.23 (s, $1 \mathrm{H}, \mathrm{NH}), 5.56$ (bs, $\left.2 \mathrm{H}, \mathrm{NH}_{2}\right), 2.54\left(\mathrm{~s}, 3 \mathrm{H}, \mathrm{CH}_{3}\right)$. ${ }^{13} \mathrm{C} \mathrm{NMR}\left(76 \mathrm{MHz}, \mathrm{CDCl}_{3}\right) \delta_{\mathrm{ppm}} 159.7,158.7,153.2,144.3,138.8$, $136.0,135.9$, 134.2, 132.3, 130.8, 130.4, 130.6, 129.9, 128.6, 127.4, 127.2 , 120.6, 116.34, 111.8, 90.0, 21.7. TOF-HRMS (EI): $\mathrm{m} /$ $z(\mathrm{M})^{+}+1$ calcd for $\mathrm{C}_{25} \mathrm{H}_{19} \mathrm{ClN}_{4} \mathrm{O}_{2} \mathrm{~S}$ 475.0917; found 475.0797 .

$\mathrm{N}$-(4-(6-Amino-5-cyano-4-(thiophen-2-yl)pyridin-2-yl)phenyl)4-methylbenzenesulfonamide (11). MP $=191-192{ }^{\circ} \mathrm{C},{ }^{1} \mathrm{H}$ NMR $\left(301 \mathrm{MHz}, \mathrm{CDCl}_{3}\right) \delta_{\text {ppm }} 8.05-8.01(\mathrm{~m}, 3 \mathrm{H}$, aromatic), $7.90(\mathrm{~d}, 2 \mathrm{H}$, $J=9 \mathrm{~Hz}$, aromatic), 7.69 (d, $1 \mathrm{H}, J=6 \mathrm{~Hz}$, aromatic), 7.42-7.33 $(\mathrm{m}, 7 \mathrm{H}$, aromatic and $\mathrm{NH}), 5.55\left(\mathrm{bs}, 2 \mathrm{H}, \mathrm{NH}_{2}\right), 2.54\left(\mathrm{~s}, 3 \mathrm{H}, \mathrm{CH}_{3}\right)$. ${ }^{13} \mathrm{C} \mathrm{NMR}\left(76 \mathrm{MHz}, \mathrm{CDCl}_{3}\right) \delta_{\mathrm{ppm}} 160.7,159.0,146.6,144.3,138.7$,
138.3, 136.1, 134.3, 129.9, 128.9, 128.7, 128.6, 128.5, 128.3, 127.4, 120.6, 117.6, 109.7, 86.0, 21.6.

$N, N^{\prime}$-((1,4-Phenylenebis(6-amino-5-cyanopyridine-4,2-diyl)) bis(4,1-phenylene))bis(4-methyl benzenesulfonamide) (1m). $\mathrm{MP}=205-208^{\circ} \mathrm{C}$, FT-IR $\left(\mathrm{KBr}, \nu, \mathrm{cm}^{-1}\right): 3506-3388,3272,3000$, 2225, 1630, 1576, 1344, 1162. ${ }^{1} \mathrm{H}$ NMR (301 MHz, $\left.\mathrm{CDCl}_{3}\right) \delta_{\mathrm{ppm}}$ 10.63 (bs, 2H, NH), 8.04 (dd, $2 \mathrm{H}, J=8.9,2.5 \mathrm{~Hz}$, aromatic), 7.837.70 (m, 9H, aromatic), 7.59-7.56 (m, 3H, aromatic), 7.41-7.38 (m, $4 \mathrm{H}$, aromatic), 7.23 (dd, $4 \mathrm{H}, J=8.4,1.8 \mathrm{~Hz}$, aromatic), 7.01 $\left(\mathrm{s}, 2 \mathrm{H}, \mathrm{NH}_{2}\right), 6.84-6.71\left(\mathrm{~m}, 4 \mathrm{H}\right.$, aromatic and $\left.\mathrm{NH}_{2}\right), 2.36(\mathrm{~s}, 3 \mathrm{H}$, $\mathrm{CH}_{3}$ ), 2.35 (s, 3H, $\mathrm{CH}_{3}$ ). TOF-HRMS (EI): $\mathrm{m} / \mathrm{z}(\mathrm{M})^{+}+1$ calcd for $\mathrm{C}_{44} \mathrm{H}_{34} \mathrm{~N}_{8} \mathrm{O}_{4} \mathrm{~S}_{2}$ 803.2144; found 803.5102.

\section{Conflicts of interest}

There are no conflicts to declare.

\section{Acknowledgements}

We thank the Bu-Ali Sina University and Iran National Science Foundation [(INSF), grant number: 98001912)] for financial support to our research group.

\section{References}

1 S. Khazalpour, M. Yarie, E. Kianpour, A. Amani, S. Asadabadi, J. Yousefi Seyf, M. Rezaeivala, S. Azizian and M. A. Zolfigol, J. Iran. Chem. Soc., 2020, 17, 1775.

2 R. Hayes, G. G. Warr and R. Atkin, Chem. Rev., 2015, 115, 6357.

3 G. Chatel and D. R. Macfarlane, Chem. Soc. Rev., 2014, 43, 8132.

4 K. Dong, X. Liu, H. Dong, X. Zhang and S. Zhang, Chem. Rev., 2017, 117, 6636.

5 (a) T. Welton, Chem. Rev., 1999, 99, 2071; (b) L. B. Abdulrauf, A. Lawal, A. Y. Sirhan and G. H. Tan, Chromatographia, 2020, 83, 11; (c) S. K. Singh and A. W. Savoy, J. Mol. Liq., 2020, 297, 112038; (d) M. Bielejewski, M. Ghorbani, M. A. Zolfigol, J. Tritt-Goc, S. Noura, M. Narimani and M. Oftadeh, RSC $A d v .$, 2016, 6, 108896.

6 F. R. Moghadam, S. Azizian, M. Bayat, M. Yarie, E. Kianpour and M. A. Zolfigol, Fuel, 2017, 208, 214.

7 M. Kharazi, J. Saien, M. Yarie and M. A. Zolfigol, J. Pet. Sci. Eng., 2020, 195, 107543.

8 (a) J. R. Naira, F. Colò, A. Kazzazib, M. Moreno, D. Bresser, R. Line, F. Bella, G. Meligrana, S. Fantini, E. Simonetti, G. B. Appetecchi, S. Passerini and C. Gerbaldi, J. Power Sources, 2019, 412, 398; (b) A. Dokouzis, F. Bella, K. Theodosiou, C. Gerbaldi and G. Leftheriotis, Mater. Today Energy, 2020, 15, 100365.

9 A. Scalia, F. Bella, A. Lamberti, C. Gerbaldi and E. Tresso, Enery, 2019, 166, 789.

10 B. A. Voss, R. D. Noble and D. L. Gin, Chem. Mater., 2012, 24, 1174.

11 M. E. Mahmoud, M. M. Osman, A. A. Yakout and A. M. Abdelfattah, J. Mol. Liq., 2018, 266, 834. 
12 Z. He and P. Alexandridis, Adv. Colloid Interface Sci., 2017, 244, 54.

13 W. Qian, J. Texter and F. Yan, Chem. Soc. Rev., 2017, 46, 1124.

14 B. Wang, L. Qin, T. Mu, Z. Xue and G. Gao, Chem. Rev., 2017, 117, 7113.

15 (a) S. Zhang, J. Zhang, Y. Zhang and Y. Deng, Chem. Rev., 2017, 117, 6755; (b) J. Nowicki, M. Muszynski and J.-P. Mikkola, RSC Adv., 2016, 6, 9194.

16 (a) S. Yotphan, L. Sumunnee, D. Beukeaw, C. Buathongjan and V. Reutrakul, Org. Biomol. Chem., 2016, 14, 590; (b) Z. Liu, A. Ebadi, M. Toughani, N. Mert and E. Vessally, $R S C$ $A d v .$, 2020, 10, 37299.

17 R. A. Azzam, R. E. Elsayed and G. H. Elgemeie, ACS Omega, 2020, 5, 10401.

18 H. S. Ibrahim, W. M. Eldehna, H. A. Abdel-Aziz, M. M. Elaasser and M. M. Abdel-Azizm, Eur. J. Med. Chem., 2014, 85, 480.

19 M. Krasavin, A. Shetnev, T. Sharonova, S. Baykov, T. Tuccinardi, S. Kalinin and C. T. Supuran, Bioorg. Chem., 2018, 76, 88.

20 Y. Dong, J. Chen and H. Xu, Chem. Commun., 2019, 55, 2027.

21 S. Bano, K. Javed, S. Ahmad, I. G. Rathish, S. Singh and M. S. Alam, Eur. J. Med. Chem., 2011, 46, 5763.

22 A. K. Ganguly, S. S. Alluri, D. Caroccia, D. Biswas, C. H. Wang, E. Kang and V. Munshi, J. Med. Chem., 2011, 54, 7176.

23 N. Siddiqui, S. N. Pandeya, S. A. Khan, J. Stables, A. Rana, M. Alam and M. A. Bhat, Bioorg. Med. Chem. Lett., 2007, 17, 255.

24 C. T. Supuran, A. Casini and A. Scozzafava, Med. Res. Rev., 2003, 23, 535.

25 M. Banerjee, A. Poddar, G. Mitra, A. Surolia and T. B. Owa, J. Med. Chem., 2005, 48, 547.

26 C. Allais, J. M. Grassot, J. Rodriguez and T. Constantieux, Chem. Rev., 2014, 114, 10829.

27 M. D. Hill, Chem.-Eur. J., 2010, 16, 12052.

28 M. Baumann and I. R. Baxendale, Beilstein J. Org. Chem., 2013, 9, 2265.

29 J. A. Bull, J. J. Mousseau, G. Pelletier and A. B. Charette, Chem. Rev., 2012, 112, 2642.

30 N. De Rycke, F. Couty and O. R. David, Chem.-Eur. J., 2011, 17, 12852.

31 G. C. Fu, Acc. Chem. Res., 2004, 37, 542.

32 N. G. Kang, M. Changez and J. S. Lee, Macromolecules, 2007, 40, 8553.

33 R. Makiura, S. Motoyama, Y. Umemura, H. Yamanaka, O. Sakata and H. Kitagawa, Nat. Mater., 2010, 9, 565.

34 W. Zhang, Y. Chen, W. Chen, Z. Liu and Z. Li, J. Agric. Food Chem., 2010, 58, 6296.

35 J. C. Xiang, M. Wang, Y. Cheng and A. X. Wu, Org. Lett., 2016, 18, 24.

36 M. A. Gouda, M. A. Berghot, G. E. Abd El Ghani and A. E. G. M. Khalil, Synth. Commun., 2014, 44, 297.

37 A. Maleki, H. Movahed and P. Ravaghi, Carbohydr. Polym., 2017, 156, 259.

38 J. F. Zhou, Y. Z. Song, J. S. Lv, G. X. Gong and S. Tu, Synth. Commun., 2009, 39, 1443.
39 M. A. Radwan, F. M. Alminderej and H. M. Awad, Molecules, 2020, 25, 255.

40 M. Muthu, R. V. Priya, A. I. Almansour, R. S. Kumar and R. R. Kumar, Beilstein J. Org. Chem., 2018, 14, 2907.

41 T. A. Abdallah, J. Heterocycl. Chem., 2007, 44, 961.

42 Y. Hao, X. P. Xu, T. Chen, L. L. Zhao and S. J. Ji, Org. Biomol. Chem., 2012, 10, 724.

43 J. F. Zhou, Y. Z. Song, J. S. Lv, G. X. Gong and S. Tu, Synth. Commun., 2009, 39, 1443.

44 M. Ghashang, K. Aswin and S. S. Mansoor, Res. Chem. Intermed., 2014, 40, 1135.

45 A. Maleki, H. Movahed and P. Ravaghi, Carbohydr. Polym., 2017, 156, 259.

46 S. K. Krishnammagari, S. G. Balwe, J. S. Kim, K. T. Lim and Y. T. Jeong, Monatsh. Chem., 2019, 150, 691.

47 N. A. Abdel-Latif, Sci. Pharm., 2005, 73, 193.

48 D. Khalili, Tetrahedron Lett., 2016, 57, 1721.

49 F. Tamaddon, S. Ghazi and M. R. Noorbala, J. Mol. Catal. B: Enzym., 2016, 127, 89.

50 A. Yahyazadeh, E. Abbaspour-Gilandeh and M. AghaeiHashjin, Catal. Lett., 2018, 148, 1254.

51 M. M. Heravi, S. Y. S. Beheshtiha, M. Dehghani and N. Hosseintash, J. Iran. Chem. Soc., 2015, 12, 2075.

52 J. F. Allochio Filho, B. C. Lemos, A. S. de Souza, S. Pinheiro and S. J. Greco, Tetrahedron, 2017, 73, 6977.

53 S. Zhi, X. Ma and W. Zhang, Org. Biomol. Chem., 2019, 17, 7632.

54 B. H. Rotstein, S. Zaretsky, V. Rai and A. K. Yudin, Chem. Rev., 2014, 114, 8323.

55 L. Levi and T. J. J. Müller, Chem. Soc. Rev., 2016, 45, 2825.

56 T. Zarganes-Tzitzikas, A. L. Chandgude and A. Dömling, Chem. Rec., 2015, 15, 981.

57 J. T. Edward, Chem. Ind., 1955, 3, 1102.

58 J. M. Erhardt and J. D. Wuest, J. Am. Chem. Soc., 1980, 102, 6363.

59 E. Juaristi and G. Cuevas, Tetrahedron, 1992, 48, 5019.

60 S. A. Glover, Tetrahedron, 1998, 54, 7229.

61 S. A. Glover, A. A. Rosser, A. A. Taherpour and B. W. Greatrex, Aust. J. Chem., 2014, 67, 507.

62 S. A. Glover and A. A. Rosser, J. Phys. Org. Chem., 2015, 28, 215.

63 R. A. Jones, A. R. Katritzky and M. Snarey, J. Turk. Chem. Soc., Sect. B, 1970, 135.

64 (a) I. V. Alabugin, Stereoelectronic Effects. A Bridge Between Structure and Reactivity. 2016; (b) I. V. Alabugin, G. , P. Gomes and M. Abdo, Comput. Mol. Sci., 2018, 9, e1389; (c) I. V. Alabugin, K. M. Gilmore and P. W. Peterson, Comput. Mol. Sci., 2011, 1, 109; (d) S. Z. Vatsadze, Y. D. Loginova, G. dos Passos Gomes and I. V. Alabugin, Chem.-Eur. J., 2017, 23, 3225.

65 (a) A. R. Katritzky, P. J. Steel and S. N. Denisenko, Tetrahedron, 2001, 57, 3309; (b) A. Nowacki and B. Liberek, Carbohydr. Res., 2018, 462, 13; (c) D. P. Curran and Y. G. Suh, Carbohydr. Res., 1987, 171, 161; (d) M. D. Drew, M. C. Wall and J. T. Kim, Tetrahedron Lett., 2012, 53, 2833; (e) S. E. Denmark, M. S. Dappen, N. L. Sear and R. T. Jacobs, J. Am. Chem. Soc., 1990, 112, 3466; $(f)$ 
A. Nowacki, D. Walczak and B. Liberek, Carbohydr. Res., 2012, 352, 177; $(g)$ C. Jäkel and K. H. Dötz, J. Organomet. Chem., 2001, 624, 172; (h) M. Asgari and D. Nori-Shargh, Struct. Chem., 2017, 28, 1803; (i) A. Nowacki and B. Liberek, Carbohydr. Res., 2013, 371, 1.

66 D. P. Curran and Y. G. Suh, J. Am. Chem. Soc., 1984, 106, 5002.

67 F. Karimi, M. A. Zolfigol and M. Yarie, Mol. Catal., 2019, 463, 20.

68 P. Ghasemi, M. Yarie, M. A. Zolfigol, A. A. Taherpour and M. Torabi, ACS Omega, 2020, 5, 3207.

69 F. Jalili, M. Zarei, M. A. Zolfigol, S. Rostamnia and A. R. Moosavi-Zare, Microporous Mesoporous Mater., 2020, 294, 109865.

70 (a) F. Karimi, M. A. Zolfigol and M. Yarie, Mol. Catal., 2020, 497, 111201; (b) S. Noura, M. Ghorbani, M. A. Zolfigol, M. Narimani, M. Yarie and M. Oftadeh, J. Mol. Liq., 2018, 271, 778 .
71 F. Karimi, M. Yarie and M. A. Zolfigol, RSC Adv., 2020, 10, 25828.

72 M. Yarie, Iran. J. Catal., 2017, 7, 85.

73 M. Yarie, Iran. J. Catal., 2020, 10, 79.

74 G. Tabacchi, M. A. Vanoni, A. Gamba and E. Fois, ChemPhysChem, 2007, 8, 1283.

75 G. Hamasaka, H. Tsuji and Y. Uozumi, Synlett, 2015, 26, 2037.

76 T. He, R. Shi, Y. Gong, G. Jiang, M. Liu, S. Qian and Z. Wang, Synlett, 2016, 27, 1864.

77 C. B. Bai, N. X. Wang, Y. Xing and X. W. Lan, Synlett, 2017, 28, 402 .

78 X. Zhao, J. Xiaoa and W. Tang, Synthesis, 2017, 49, 3157.

79 A. El-Faham, K. A. Dahlous, Z. A. AL Othman, H. A. AlLohedan and G. A. El-Mahdy, Molecules, 2016, 21, 436.

80 E. J. Kim, H. W. Ryu, M. J. Curtis-Long, J. Han, J. Y. Kim, J. K. Cho, D. Kang and K. H. Park, Bioorg. Med. Chem. Lett., 2010, 20, 4237. 